\title{
Spatiotemporal Evolution of Functional Structure of Urban Agglomeration in Central Yunnan
}

\author{
Ziye Liu*, Miaoxin Liu, Xin Chen, Xinyang Dai, and Yulin Zhu \\ International Business School, Yunnan University of Finance and Economics, Kunming, Yunnan, \\ China
}

\begin{abstract}
This paper selects data related to each representative industry in the Central Yunnan Urban Agglomeration from 2010-2019 as the research sample, and analyzes the functional structure of the Central Yunnan Urban Agglomeration through spatial Gini coefficient, primacy and location quotient. The research results show that: the economic development level of Kunming, a city with high primacy, is insufficient. Moreover, the development differences within the Central Yunnan Urban Agglomeration are large enough and it's hard to drive economic recovery. The industrial agglomeration of the Central Yunnan Urban Agglomeration is not high. There are fluctuating changes in industries that depend on the natural environment, and the epidemic has a large impact on the pillar industries as well. There are overlapping industrial functions within the Central Yunnan Urban Agglomeration. The complementarity of each function is not high enough, and the degree of regional economic integration is not enough, which is not conducive to economic recovery.
\end{abstract}

\section{Introduction}

Since the 21st century, with the development of economic globalization and the increasingly close relationship between countries and regions, the role played by urban agglomerations in the national development system has become increasingly important. The development of the Central Yunnan Urban Agglomeration, as the main carrier of the economic region of Yunnan Province, has not been satisfactory in recent years. Part of the reason for this slow development is the irrational layout of industries and the lack of synergy mechanisms between cities, which has resulted in the crowding out of resources. The functional planning of the Central Yunnan Urban Agglomeration therefore requires a more precise analysis of the division of functions between the cities and an improved development strategy for the urban agglomeration, in order to address the problems in the functional structure.

Therefore, this paper proposes to study the evolution of the spatial and temporal differentiation of the functional structure of the Central Yunnan Urban Agglomeration

\footnotetext{
* Corresponding author: 876054340@qq.com
} 
(consisting of Kunming, Qujing, Yuxi, Chuxiong and seven cities of Honghe), using three methods: spatial Gini coefficient, primacy and location quotient. The marginal contributions of this study are: this paper will analyse the data related to each sector of the Central Yunnan Urban Agglomeration in both temporal and spatial dimensions, with the ultimate aim of promoting the optimisation of urban functions in the Central Yunnan Urban Agglomeration, and providing data support and theoretical basis for the adjustment of the functional structure of the Central Yunnan Urban Agglomeration.

\section{Literature Review}

Existing studies mostly classify cities or industries at the macro level. While at the micro level, they mostly measure the degree of the specialization of a single industry with the objective of industrial transformation and upgrading, so as to make predictions on the development trend of a single industry. For example, Ullman (1957) argued that there is a process of interaction between cities according to the division of urban functions in urban agglomerations. The cities with strong basic urban functions can export to cities with only non-basic functions, achieving coordinated development between regions [1]. On the other hand, Friedman (1963) had its definition of urban markets as well as urban system hierarchy network [2]. In terms of research methodology, Nelsen (1955) used statistical analysis and mean-standard deviation classification to classify American cities [3]. Lao, Zhang, Shen and Wang (2017) used cluster analysis to classify the functions of the middle reaches of Yangtze River Urban Agglomeration [4]. Zeng and Fang (2020) used spatial autocorrelation analysis to measure the aggregation degree of logistics industry in Guangdong Province [5]. Zeng, Li, Xing and Hu (2020) used city primacy to measure 19 urban agglomerations in China and analysed the agglomeration and diffusion effects of central cities [6]. After the system of urban agglomerations and their functional structure was gradually improved, some scholars began to analyse different levels of the functional structure of urban agglomerations in different directions. De Groot, Poot and Smit (2016) analysed specialisation, diversity, and competition through MAR externalities, Porter externalities and Jacobs externalities [7]. Ma and Zhao (2019) studied the spatial layout characteristics and industrial structure evolution of the Harbin-Changchun urban agglomeration, and the spatial correlation between industrial organization, and analyzed urban agglomeration as well [8].

Based on existing research, this paper collects and measures data on the major industries of the cities in the Central Yunnan Urban Agglomeration, comparing and analysing the data obtained, so as to make predictions on the development trends of industries and urban agglomerations, and to put forward feasible suggestions for the adjustment and development of the urban function structure of the Central Yunnan Urban Agglomeration.

\section{Research Design}

\subsection{Data sample selection and sources}

The research object of this paper is the Central Yunnan Urban Agglomeration. The data used for each city are sourced from the Yunnan Statistical Yearbook from 2010-2019, mainly including the number of people employed in eight industries in cities from both the Central Yunnan Urban Agglomeration and Yunnan Province, the total employment in cities from both the Central Yunnan Urban Agglomeration and Yunnan Province, the population in cities from both the Central Yunnan Urban Agglomeration and Yunnan Province, and GDP in cities from both the Central Yunnan Urban Agglomeration and Yunnan Province. 


\subsection{Model Specification}

In order to better reflect the characteristics of the functional structure of the Central Yunnan Urban Agglomeration and the trend of spatial and temporal divergence, three models are used in this study to build the research process: Firstly, the spatial Gini coefficient formula is used to measure the spatial Gini coefficients of the major industries in the Central Yunnan Urban Agglomeration and the five cities/autonomous prefectures it contains, and the data is visualised in a graph. The spatial Gini coefficient is calculated as follows:

$$
\mathrm{G}=\sum_{\mathrm{i}=1}^{\mathrm{n}}\left(\mathrm{S}_{\mathrm{i}}-\mathrm{X}_{\mathrm{i}}\right)^{2}
$$

Where: $G$ is the spatial Gini coefficient of the industry, $S_{i}$ is the proportion of employment in a particular industry (i.e. flowers, seedlings, construction, etc.) in the region $i$ to employment in that industry in the province, and $X_{i}$ is the proportion of employment in that region to total employment in the province.

Secondly, this paper uses economic primacy and city primacy to reflect the economic scale structure, city scale structure and population concentration of the Central Yunnan Urban Agglomeration. It is generally believed that a city's degree of primacy is less than 2, indicating a normal structure and appropriate concentration; the degree is greater than 2 , indecating there is a tendency of structural imbalance and over-concentration. The calculation formula is as follows:

$$
\begin{aligned}
& \mathrm{S}_{\mathrm{t}}=\frac{\mathrm{G}}{\mathrm{G}_{\mathrm{t}}} \\
& \mathrm{S}_{\mathrm{i}}=\frac{\mathrm{P}}{\mathrm{P}_{\mathrm{i}}}
\end{aligned}
$$

Where: $S_{t}$ is the economic primacy of city $t, G$ is the economic scale (GDP) in Kunming, and $G_{t}$ is the economic scale of city $t$.

$\mathrm{S}_{\mathrm{i}}$ is the city primacy of city $\mathrm{i}, \mathrm{P}$ is the population scale of Kunming, the first city in the Central Yunnan Urban Agglomeration, $P_{i}$ is the population size of city $i$.

Finally, this study applies the location quotient to measure the degree of development, specialisation and the economic support base that each city in the Central Yunnan Urban Agglomeration can bring to the region by industry. It is calculated as follows:

$$
\mathrm{Lq}_{\mathrm{ij}}=\frac{\mathrm{G}_{\mathrm{ij}} / \mathrm{G}_{\mathrm{i}}}{\mathrm{G}_{\mathrm{j}} / \mathrm{G}}
$$

Where: $\mathrm{Lq}_{\mathrm{ij}}$ is the location quotient in industry $\mathrm{j}$ in city $\mathrm{i} ; \mathrm{G}_{\mathrm{ij}}$ is the number of workers in industry $j$ in city $i ; G_{i}$ is the number of workers in city $i ; G_{j}$ is the number of workers in industry $j$ in Yunnan Province; $G$ is the number of workers in Yunnan Province.

\section{Analysis Of Data Results}

\subsection{Based on the spatial Gini coefficient}

Using Model (1), the spatial Gini coefficients for each industry in the Central Yunnan Urban Agglomeration and the five cities/autonomous prefectures can be obtained for the decade 2010-2019.

On the whole, the spatial Gini coefficients of all industies in the urban agglomeration of central Yunnan Province in China are mostly on a stable trend. Meanwhile, the spatial Gini coefficients of the health, social security, wholesale and retail, accommodation and catering, manufacturing and construction industries all remain at a low value year-round.

At the same time, it can be found from Fig.1 that the main industries with high spatial Gini coefficients among the various industries in the Central Yunnan Urban Agglomeration 
are mining and agriculture, forestry and fishery. Overall, the spatial Gini coefficient of the mining industry is perennially at the highest value among all industries and is the main industry for the development of the Central Yunnan Urban Agglomeration. But its value fluctuates widely and could be closely related to the discovery or depletion of mineral resources. Secondly, the spatial Gini coefficient of agriculture, forestry, animal husbandry and fishery is perennially in the second position in the Central Yunnan Urban Agglomeration, but its value declined steeply in 2017-2019, which may be related to the significant decrease of resources for agriculture, forestry, animal husbandry and fishery in the Central Yunnan Urban Agglomeration. On the other hand, the spatial Gini coefficient of the electricity, gas and water production and supply industry in the Central Yunnan Urban Agglomeration has been in a low state from 2010-2019, but its value suddenly increased in 2019. The situation may be due to the remarkable process Kunming has achieved in advancing this aspect of the electricity pillar industry in Yunnan Province, mainly hydropower, which has led to a large demand for employment in this industry, and therefore a sharp increase in employment driving the spatial Gini coefficient higher [9].

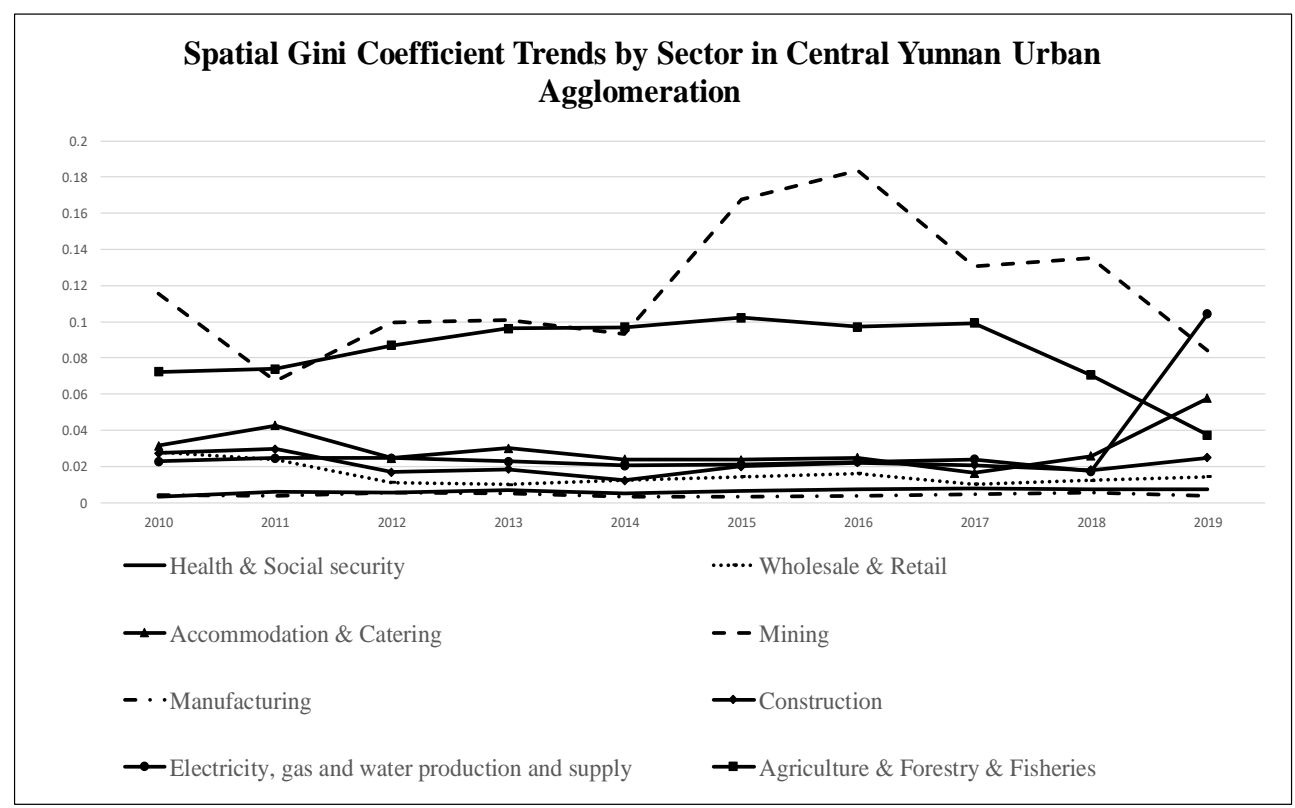

Fig. 1. Spatial Gini coefficients trends by industry in Central Yunnan Urban Agglomeration.

The analysis above shows that in the past decade, mining and agriculture, forestry, animal husbandry and fishery industries have played a pivotal role in the development of the Central Yunnan Urban Agglomeration, relying on a high degree of spatial aggregation and a relatively mature industrial system to achieve the efficient deployment of production factors and the rational use of resources. However, the mining and agriculture, forestry and fishery industries are both extremely dependent on natural resources with non-renewable characteristics, indicating that there will be significant bottlenecks in these two industries. According to the calculation results, the degree of concentration of these two industries in the Central Yunnan Urban Agglomeration has shown a decreasing trend from 2016 to 2019. It indicates that some capital and labour have already withdrawn from these two industries, which greatly reduces the long-term dependence of the Central Yunnan Urban Agglomeration on these two industries. This greatly reduces the possibility of the Central Yunnan Urban Agglomeration relying on these two industries for long-term development. 
Therefore, there is a need to adjust the current state of the industrial structure of the Central Yunnan Urban Agglomeration. Firstly, the development patterns of mining and agriculture, forestry and fishery need to be adjusted. As once leading industries, the two are important components of the industrial structure of the Central Yunnan Urban Agglomeration. So rational planning of resources, reducing the proportion of the two in the industrial structure and providing some appropriate protection policies for the relevant resources are planed not only for the long-term development of the industries, but also in helping to buffer the impact of industrial restructuring. Secondly, there is a necessity to find new industries that can be developed in the long term. In terms of data, the concentration of the electricity, gas and water production and supply industry in the Central Yunnan Urban Agglomeration has increased significantly, while there is a decline in the concentration of the mining and agriculture, forestry and fishery industries, from which I could interpret as a subtle shift in the market. In essence, these three industries are all dependent on natural resources, but the biggest difference between the electricity, gas and water production and supply industry and the first two is that the resources they rely on are highly renewable, which means that they have the potential to develop as dominant industries in the long term. At the same time, the fact that it has the same origin as the first two industries can help it to absorb the capital and labour lost by the first two industries, which is a very important advantage over other industries. It is also an important opportunity for the Central Yunnan Urban Agglomeration to adjust its industrial structure for sustainable development on its own.

\subsection{Based on primacy}

\subsubsection{Economic primacy}

By substituting the relevant data into model (2), Table 1 was obtained.

Table 1. Primacy (economy).

\begin{tabular}{|c|c|c|c|c|c|c|c|c|c|c|}
\hline Year & 2010 & 2011 & 2012 & 2013 & 2014 & 2015 & 2016 & 2017 & 2018 & 2019 \\
\hline Primacy(Economy) & 1.0986 & 1.0995 & 1.1006 & 1.1013 & 1.1027 & 1.1042 & 1.1059 & 1.1080 & 1.1128 & 1.1250 \\
\hline
\end{tabular}

According to Table 1, the economic primacy value of the Central Yunnan Urban Agglomeration, represented by Kunming City, increased slowly from 2010 to 2019, and reached a maximum value of 1.125 , which is less than 2 in 2019. It indicates that the economic development structure of the Central Yunnan Urban Agglomeration is normal and the degree of industrial agglomeration is not high. The highest value is less than 1.5, which indicates that Kunming's economic development is average. But as the first city, Kunming has weak economic impetus to the Central Yunnan Urban Agglomeration and may not be able to drive the development of the whole region. From the analysis ablove, it can be recognized that the scale distribution of the Central Yunnan Urban Agglomeration belongs to the moderate first place distribution of the first-place distribution type. The city scale hierarchy is unbalanced, and the first place of the central city needs to improve the city scale structure. Break the single mode of expanding the city population, and instead attract advantageous industries and high-quality talents to further enhance and strengthen regional agglomeration and lead the functional development of the surrounding cities. 


\subsubsection{City primacy}

By substituting the relevant data into model (3), Table 2 was obtained.

In recent years, due to the export constraints, national economic strategies have gradually tended towards endogenous growth. Based on this situation, the economic drive of the country's top city on the surrounding urban agglomerations is particularly important. According to Table 2, the city scale primacy of cities in Central Yunnan represented by Kunming fluctuates from 2010 to 2019 , but the overall average is greater than 2. This indicates that the cities in Central Yunnan represented by Kunming are the gathering place of talents and have higher economic vitality. But it also shows that the development of population scale in Central Yunnan is uneven, with large differences in local development.

Table 2. Primacy (population).

\begin{tabular}{|c|c|c|c|c|c|c|c|c|c|c|}
\hline Year & 2010 & 2011 & 2012 & 2013 & 2014 & 2015 & 2016 & 2017 & 2018 & 2019 \\
\hline Primacy(population) & 2.1086 & 2.0742 & 2.1505 & 2.1562 & 2.3979 & 2.0948 & 2.4226 & 2.5025 & 2.5862 & 2.4552 \\
\hline
\end{tabular}

At the same time, the spatial structure of the Central Yunnan Urban Agglomeration has been profoundly altered by the interaction of various factors. In the Central Yunnan Urban Agglomeration, Kunming, the first place of the central city, has fluctuated over the decade, but it is still at its highest level. As the large-scale development of Kunming, the cost from the surrounding provinces and regions is far less in terms of resources than in terms of economic-driven benefits. It slows down the predatory effect of the first city as well. Therefore, the development of the first city is still beneficial to the economic growth of the nearby provinces and the gradual formation of a high spatial-geographical concentration of the economies of the various provinces will lead to an increase in the degree of openness to the outside world, which will help to promote the development of the Central Yunnan Urban Agglomeration as a whole.

\subsection{Based on location quotient}

\subsubsection{Analysis of the urban location quotient of the Central Yunnan Urban Agglomeration from an industry perspective}

By substituting the relevant data into model (4), Table 3 was obtained.

On the whole, Kunming has a relatively balanced overall development, except for heavy industry, and exceeds the other sub-centres; Qujing and Yuxi have a relatively stronger development; and the overall level of Chuxiong Prefecture needs to be improved.

The city with the highest level of specialisation and agglomeration in the construction industry is Kunming, which remains at a high level overall. As the Wujiaba area in Kunming has undergone large-scale renovation and construction of a large urban central park in recent years, it attracts a large number of real estate industries [10]. At the the same time, the real estate and construction industries are cooperative parties, so the renovation of this area has had a positive impact on the construction industry in Kunming in some ways. The two cities with a relatively low level of specialisation in the industry and relatively large fluctuations in development are Yuxi and Chuxiong. In 2011, the two cities reached the trough of the construction industry in each city from 2010 to 2015 at the same time. Generally speaking, the two cities are fluctuating on the verge of reaching a basic level for the local demand.

Agriculture, forestry, and fishery have a low degree of specialisation in the Central Yunnan Urban Agglomeration, with no city location quotient reaching 1 in any year. The 
highest and lowest levels of specialisation are in Chuxiong and Kunming in comparison. In terms of agriculture, although Yunnan is a traditional agricultural province, the agriculture, forestry and fishery industry is not prime and dominant in the Central Yunnan Urban Agglomeration. The main reason is that some places of Yunnan's specialities, such as Lincang for pu-erh tea, Yuanmou County for high quality vegetables and Dehong for small grain coffee beans, are not the part of the Central Yunnan Urban Agglomeration. The Central Yunnan Urban Agglomeration is not dominated by modern or traditional agriculture.

Table 3. Location quotient of the Central Yunnan Urban Agglomeration in 2010 and 2019.

\begin{tabular}{|c|c|c|c|c|c|c|c|c|}
\hline City & (A) & (B) & (C) & (D) & (E) & (F) & (G) & (H) \\
\hline \multirow{2}{*}{ Kunming } & 0.83 & 1.48 & 1.53 & 0.3 & 1.11 & 1.52 & 0.54 & 0.16 \\
\hline & 0.74 & 1.35 & 1.72 & 0.43 & 1.02 & 1.48 & 1.99 & 0.41 \\
\hline Difference & -0.09 & -0.13 & 0.19 & 0.13 & -0.09 & -0.04 & 1.45 & 0.25 \\
\hline \multirow{2}{*}{ Qujing } & 0.75 & 0.54 & 0.32 & 3.58 & 1.27 & 0.74 & 1.32 & 0.36 \\
\hline & 0.94 & 0.87 & 0.4 & 3.29 & 1.41 & 1.35 & 0.63 & 0.78 \\
\hline Difference & 0.19 & 0.33 & 0.08 & -0.29 & 0.14 & 0.61 & -0.69 & 0.42 \\
\hline \multirow[b]{2}{*}{ Yuxi } & 69 & 106 & 085 & & 156 & 0 & 106 & 034 \\
\hline & 1.09 & 1.76 & $\begin{array}{l}0.05 \\
0.64 \\
\end{array}$ & $\begin{array}{l}1.55 \\
1.4 \\
\end{array}$ & $\begin{array}{l}1.50 \\
1.19 \\
\end{array}$ & $\begin{array}{l}0.49 \\
0.66 \\
\end{array}$ & $\begin{array}{l}1.00 \\
0.33\end{array}$ & 0.54 \\
\hline Difference & -0.03 & -0.2 & -0.21 & 0.05 & -0.37 & 0.17 & -0.73 & 0.18 \\
\hline & & & & & & & & \\
\hline \multirow{2}{*}{ Chuxiong } & 1.17 & 0.76 & 0.65 & 1.32 & 0.82 & 1.13 & 0.91 & 0.48 \\
\hline & 1.09 & 0.67 & 0.46 & 1.11 & 0.94 & 1.05 & 0.26 & 0.38 \\
\hline Difference & -0.08 & -0.09 & -0.19 & -0.21 & 0.12 & -0.08 & -0.65 & -0.1 \\
\hline
\end{tabular}

Note: (A): Health \& Social security; (B): Wholesale \& Retail; (C): Accommodation \& Catering; (D): Mining; (E): Manufacturing; (F): Construction; (G): Electricity, gas and water production and supply; $(\mathrm{H})$ : Agriculture \& Forestry \& Fisheries;

\subsubsection{Analysis of the complementarity of functional structure sectors from the perspective of urban agglomerations}

Although there are minor changes in the mainstream industries of the cities, but in general, the mainstream industries of the cities do not vary significantly and the functional structure of the Central Yunnan Urban Agglomeration is relatively stable. We have analysed the mainstream industries in each city with a frequency greater than $50 \%$ in each year as the mainstream industries in the Central Yunnan Urban Agglomeration over the past 10 years, and plotted the following table:

Table 4. Analysis of mainstream industries in Central Yunnan Urban Agglomeration.

\begin{tabular}{|l|c|c|c|c|c|c|c|c|}
\hline \multicolumn{1}{|c|}{ City } & (A) & (B) & (C) & (D) & (E) & (F) & (G) & (H) \\
\hline Kunming & & $\checkmark$ & $\checkmark$ & & $\checkmark$ & $\checkmark$ & & \\
\hline Qujing & & & & $\checkmark$ & $\checkmark$ & $\checkmark$ & $\checkmark$ & \\
\hline Yuxi & & $\checkmark$ & & & $\checkmark$ & & & \\
\hline Chuxiong & $\checkmark$ & & & $\checkmark$ & & & $\checkmark$ & \\
\hline
\end{tabular}


The highest industry complementarities are in the health and social security, accommodation and catering. In terms of the health and social security, Chuxiong can export industries to Kunming, Qujing and Yuxi. Kunming, which is the most prosperous and commercial in the city cluster, has the highest concentration of accommodation and catering, which can be exported to other sub-centres, thus sharing resources.

Industries with relatively high complementarity are the wholesale and retail trade, mining, construction, and electrical, gas and water production and supply. Kunming is the central city of the Central Yunnan Urban Agglomeration, with convenient transport links for the flow of goods. In addition, Kunming and Yuxi are adjacent to each other, and it allows for the formation of a larger "Kunming-Yuxi" wholesale and retail market centre, which can better radiate the Central Yunnan Urban Agglomeration and export to surrounding cities. The mining industry and the electrical, gas and water production and supply industry are relatively highly complementary. But there is a slight overlap of urban functions in the mining industry and the electrical, gas and water production and supply industry in Qujing due to the over-concentration and high specialisation of the mining industry in Qujing. Additionally, Qujing is rich in water, gas and electricity. However, Chuxiong Prefecture is also in a position to have an advantage in similar industries within the Central Yunnan Urban Agglomeration. Among the four cities, there are three cities, Kunming, Qujing and Yuxi, having the manufacturing as the mainstream urban industry, with a large overlap and low industrial complementarity. Agriculture, forestry and fishery industry, due to the natural environment, are basically not self-sufficient in the Central Yunnan Urban Agglomeration and need to be imported from neighbouring cities in Yunnan Province.

\subsubsection{Optimising the functional structure of the Central Yunnan Urban Agglomeration}

According to the data above, although the wholesale and retail and accommodation and catering are both mainstream industries in Kunming, neither industry has exceeded a location quotient of 1.5 at the same time in the past ten years, becoming an industry with relatively high comparative advantages at the same time. Furthermore, on the whole, there is still room for development in wholesale and retail trade compared to accommodation and catering. The healthy development of the tertiary industry represented by the wholesale and retail industry and the accommodation and catering industry can balance the over-tourism of the tertiary industry in the Central Yunnan Urban Agglomeration and comprehensively coordinate the economic structure [11]. Further, the enhancement of the restaurant industry will have a positive impact on the service trade [12]. When the location quotient of both can exceed 1.5 at the same time, it not only allows the wholesale and retail industry and the accommodation and catering industry to be fully developed, but also increases the core competitiveness of Kunming as the central city of the Central Yunnan Urban Agglomeration. Increase the diversification of business models, brand building characteristics, and increase the proportion of trade. Use Kunming as a window to seek a larger international market.

According to Table 4, Kunming and Qujing each have four mainstream industries, making them the two cities with the most mainstream industries in the Central Yunnan Urban Agglomeration. However, there is an overlap in the division of urban functions in manufacturing and construction. On the other hand, the construction and manufacturing industries in the Central Yunnan Urban Agglomeration are also largely driven by Kunming and Qujing.

For Kunming City, it can turn to the investment in manufacturing industry and increase the investment in high-tech industry to alleviate the high overlap rate of manufacturing 
industry in Central Yunnan Urban Agglomeration. Additionally, the other target is to solve the problem of resources for reasonable coordination, increase the core competitiveness in the central city, improve the economic status and increase the economic radiation to Central Yunnan Urban Agglomeration.

At the same time, as Qujing City and Chuxiong Prefecture in the mining industry and electrical, gas and water production and supply industry have a certain degree of overlap, it's also necessary to reduce investment and constructio of Chuxiong Prefecture in these two industries.

\section{Conclusions}

\subsection{Integrated and comprehensive planning of the functions and positioning of the Central Yunnan Urban Agglomeration}

The optimisation of the functional structure of the cities in Central Yunnan should be planned in a comprehensive manner from the height of the province. Considering regional strengths, urban development needs, development conditions and development bases, comprehensively integrating economic, social, resource and environmental factors, the scientific and reasonable adjustments should be formulated to the industrial countermeasures of the Central Yunnan Urban Agglomeration to guide the relative transformation and development of its functional structure. Position the urban function of cities to make it coordinate and sustainable, and establish a corresponding city image. Ensure that the functions of the cities are subordinated to the general situation of healthy urban development in the province, which is conducive to the sustainable development of the regional urban economy and the improvement of people's living standards.

\subsection{Ensuring the government's macro-regulatory role over the functional structure of individual cities}

The government's macro-control should be strengthened. Firstly, consolidate measures for the stability of advantageous industries. Secondly, support the development of some industries in light of the current situation, so as to reduce the impact of the new epidemic on the social economy and even create conditions for a good multi-polar development in the future. According to the analysis based on the spatial Gini coefficient and location quotient, the relevant protection policies should be introduced for the mining industry, agriculture, forestry and fishery industriy.

\subsection{Use of market regulation function to promote industrial transfer}

Market regulation is an important means of rational allocation of resources, and industrial transfer is one of the most direct and effective means of restructuring the functions of cities. Giving full play to the regulating function of the market and promoting the reasonable transfer of the main industries in central Yunnan cities within the national region will help the industries in central Yunnan cities to construct a scientific industrial system and optimize the functional structure of the cities. As domestic production costs, especially labour costs, are increasing, the market, guided by the law of value, spontaneously regulates the operation of the economy. Labour and some capital-intensive industries are gradually shifting from the eastern coastal areas, where production costs are high, to the inland areas, where labour resources are abundant and costs are low, whch is an inevitable trend. The transfer of industries between cities in China is more active. By making full use of the 
regulating function of the market to actively guide the transfer of industries within and between cities under macro-control, the system of city functions will be more adapted to the requirements of healthy urban development.

\subsection{Improve competitiveness by highlighting the advantageous functions of the first city}

Under the background of the economic globalisation, the development of cities into a global circumstance depends not on their own shortcomings, but on the dominant conditions. Highlighting the city's advantageous functions will enable it to take its place in the global development for the big data. It is particularly important for Kunming, the first city in the Central Yunnan City Agglomeration, to highlight its own city's superior functions. From the perspective of primacy analysis, it shows that Kunming has an average level of economic development and lacks the ability to drive the development of the Central Yunnan City Agglomeration as the first city. Therefore, the Central Yunnan Urban Agglomeration needs to improve the economic primacy of Kunming as the first city in central Yunnan, and combine the industrial structure of Kunming with relevant national policies to meet the needs of Kunming's green industrial development. Relying on Kunming's resource advantages, vigorously develop Kunming's advantageous and characteristic industries, improve the economic vitality of the Central Yunnan Urban Agglomeration, and promote Kunming to achieve the "win-win" goal of ecological construction and economic development as soon as possible. It will also enable Kunming to realise the 13th Five-Year Plan as soon as possible and become an environmental and ecological home for harmonious development.

This paper is sponsored by NSFC'Study on the coupling mechanism between urban agglomeration spatial structure and economic resilience under the impact of major events', with Project Number 72063033 .

\section{References}

1. E. L. Ullman, American commodity flow (University of Washington, Seattle, 1957)

2. M. Friedman, A. J. Schwartz, A monetary history of the United States 18671960(Princeton University Press, Princeton, 1963)

3. H J. Nelson, A Service classification of American cities, Economic Geography, 31, 189-210(1955)

4. X. Lao, Y. Zhang, T. Y. Shen, M. Wang, Study on the characteristics of urban functional structure of urban agglomeration in the middle reaches of the Yangtze River, Urban Development Studies, 24, 111-117(2017)

5. Y. Zeng, Y. Fang, Prediction of agricultural products logistics demand in Guangdong Province based on grey prediction model, Journal of Shanxi Institute of Economic Management, 28, 44-48(2020)

6. P. Zeng, H. T. Li, X. Y. Xing, Y. Hu, A study on the influence of the first degree of central cities on the coordinated development of regional economy: Analysis based on nineteen large urban agglomerations in China, Journal of Chongqing University (Social Science Edition), 26, 1-16(2020)

7. H. 1. F. De Groot, J. Poot, M. J Smit, Which agglomeration externalities matter most and why? Journal of Economic Surveys, 30, 756-782(2016) 
8. C. G. Ma, T. Y. Zhao, The correlation between industrial organization and sustainable growth of city cluster spatial: A case study of Harbin-Changchun city cluster, Energy Procedia, 157, 773-781(2019)

9. J. J. Ren, Research on marketing strategy improcement of S Power Generation Company under the background of Yunnan Province new electric power sysytem reform, Master thesis from Yunnan Normal University (2021)

10. Q. Q. Xie, A study on the customer positionning evalution of project D in Wujiaba, Kunming, Master thesis from Kunming University of Science and Technology (2020)

11. J. Q. Wu, Study on the division of urban functions in Yunnan, Master thesis from Yunnan Normal University (2014)

12. X. Tian, Changes in the competitiveness of the resautant industry and its impact on trade in services, Master thesis from University of International Business and Economics (2009) 